\title{
Diagnostic value of classical and atypical antineutrophil cytoplasmic antibody (ANCA) immunofluorescence patterns
}

\author{
R C W Wong, R A Silvestrini, J A Savige, D A Fulcher, E M Benson
}

\begin{abstract}
Background-The "classical" antineutrophil cytoplasmic antibody (C-ANCA) pattern seen on indirect immunofluorescence (IIF) is characterised by granular cytoplasmic staining showing central or interlobular accentuation, and is strongly associated with antiproteinase -3 antibodies (PR3-ANCA) and Wegener's granulomatosis. However, many laboratories report C-ANCA in the presence of any cytoplasmic IIF staining, regardless of pattern, which risks reducing the diagnostic value of this pattern.
\end{abstract}

Aims-To classify different cytoplasmic ANCA patterns and thus determine whether stringent application of the classical criteria for C-ANCA would produce better correlation between C-ANCA and (1) PR3-ANCA enzyme linked immunosorbent assay (ELISA) results; (2) a diagnosis of systemic vasculitis (including Wegener's granulomatosis).

Methods-72 sera with cytoplasmic IIF collected over a two year period were analysed by IIF and a commercial PR3-ANCA ELISA kit.

Results-Three IIF patterns were defined: "classical/true" C-ANCA as described above (n = 27 (37.5\%)); "flat" ANCA with homogeneous cytoplasmic staining (n = 21 (29\%)); and "atypical" ANCA which included all other cytoplasmic patterns $(n=24(33.5 \%))$. Twenty five of the 27 true C-ANCA sera $(92.5 \%)$ contained PR3-ANCA $(p<0.0001)$, but none of the 21 with flat ANCA and only one of the 24 with atypical ANCA. From clinical data on 23 of the 27 true C-ANCA positive patients, $20(87 \%)$ had evidence of Wegener's granulomatosis or systemic vasculitis ( $\mathrm{p}<0.0001 v$ the other two patterns). However, none of 19 sera with flat ANCA and clinical data had evidence of systemic vasculitis.

Conclusions-Restricting the term "cANCA" to the "classical" description of central/interlobular accentuation on IIF, will improve its correlation with PR3ANCA positivity and a diagnosis of systemic vasculitis.

(f Clin Pathol 1999;52:124-128)

Keywords: cytoplasmic ANCA; classical ANCA; atypical ANCA

Antineutrophil cytoplasmic antibodies (ANCA) are autoantibodies directed against neutrophil cytoplasmic antigens. ${ }^{1-6}$ ANCA which produce cytoplasmic granular fluorescence showing central or interlobular accentuation on indirect immunofluorescence (IIF) using ethanol fixed normal human neutrophils ${ }^{2}$ are reported to have a high diagnostic specificity for Wegener's granulomatosis, a form of systemic vasculitis. ${ }^{3-5}$ This IIF pattern has been termed C-ANCA, "c" denoting the "classical" pattern illustrated in the original report associating ANCA with Wegener's granulomatosis, ${ }^{6}$ and correlates with antibodies to proteinase-3 (PR3), a $29 \mathrm{kDa}$ serine protease. ${ }^{17}$ PR3ANCA are detectable by enzyme linked immunosorbent assay (ELISA), and are strongly associated with Wegener's granulomatosis. ${ }^{1-8}$ Nevertheless, IIF remains the preferred screening test for ANCA by most laboratories as it is cheaper, possibly more sensitive than standard ELISA, ${ }^{9}$ and also shows other useful IIF patterns. ${ }^{1}$

Despite the above definition of C-ANCA at the First International ANCA workshop ${ }^{2}$ and by the Association of Clinical Pathologists, ${ }^{10}$ routine laboratories often report C-ANCA when there is any cytoplasmic staining regardless of pattern. A recent Australasian quality assurance programme survey found that 34 of 39 laboratories (87\%) reported the pattern shown in fig 1A as C-ANCA positive, despite the absence of central/interlobular accentuation. Only two of 20 laboratories $(10 \%)$ that performed a PR3-ANCA ELISA on this sample reported an unequivocally positive result. In addition, several recent publications and immunopathology texts have failed to emphasise the importance of central/ interlobular accentuation of cytoplasmic staining ${ }^{11-14}$ and have even used non-classical patterns to illustrate C-ANCA. ${ }^{11}$ This trend away from established guidelines ${ }^{2}{ }^{10}$ risks reducing the diagnostic usefulness of the C-ANCA IIF pattern for Wegener's granulomatosis. Observations in our laboratory have shown that PR3-ANCA negative, cytoplasmic ANCA IIF positive sera in which the staining pattern was not considered had a low positive predictive value (15\%) for Wegener's granulomatosis and systemic vasculitis (Mallon D et al, submitted for publication).

The aims of this study were to classify different cytoplasmic ANCA patterns and therefore determine whether stringent application of the above criteria ${ }^{2}{ }^{10}$ would produce a better correlation between C-ANCA and (1) PR3-ANCA ELISA results; (2) a diagnosis of systemic vasculitis (including Wegener's granulomatosis).
Accepted for publication 16 September 1998 


\section{Methods}

SPECIMENS

We studied sera obtained from 72 different patients which produced cytoplasmic IIF staining. Forty eight $(67 \%)$ of these were randomly selected from stored specimens collected over a two year period in a diagnostic immunopathology laboratory that examines about 3500 specimens for ANCA a year. Twenty four (33\%) were consecutive specimens collected over a four month period in 1996.

INDIRECT IMMUNOFLUORESCENCE

IIF was adapted from the method of Wiik. ${ }^{15}$ Briefly, human neutrophils were isolated from peripheral blood of healthy donors by layering over lymphocyte separation medium (ICN Biomedicals) for 30 minutes at $400 \mathrm{~g}$. Cells remaining above the medium were washed in phosphate buffered saline (PBS) with $1 \%$ human serum albumin (Sigma), cytocentrifuged onto slides, and fixed with ethanol at $4^{\circ} \mathrm{C}$ for five minutes. Patients' sera were applied at serial fourfold dilutions (from 1:10 to $1: 640$ ) in PBS. Bound antibodies were detected with fluorescein isothiocyanate conjugated rabbit antihuman IgG (Dako) diluted 1:60. PR3ANCA positive serum from a patient with Wegener's granulomatosis and normal human serum were used as positive and negative controls, respectively.
The IIF staining pattern was classified into one of three categories by consensus agreement between two observers (RW and RS) who did not know the PR3-ANCA ELISA results when performing the IIF: (1) "true" C-ANCA pattern (fig 1B) with central or interlobular accentuation of granular cytoplasmic staining ${ }^{210}$; (2) "flat" ANCA pattern (fig 1C) with homogeneous granular cytoplasmic staining and no central/interlobular accentuation ${ }^{10}$; and (3) "atypical" ANCA pattern (fig 1D), which included all other cytoplasmic patterns. The end point titres were determined by consensus agreement.

ELISA FOR PR3-ANCA AND BACTERICIDAL PERMEABILITY INCREASING PROTEIN (BPI) ANCA PR3-ANCA were detected using a solid phase ELISA (ORGenTec), performed according to the manufacturer's instructions. Briefly, prediluted standards, controls and patients' sera were added to PR3 coated microtitre plate wells and incubated for 30 minutes. After washing, horseradish peroxidase conjugated antihuman IgG was added, and then developed with 3,3',5,5'-tetramethyl benzidine. After 15 minutes, colour development was stopped with $1 \mathrm{M}$ hydrochloric acid and the optical density measured at $450 \mathrm{~nm}$. A six point standard curve was used, based on the company's internal reference material. A cut off value of 10 $\mathrm{U} / \mathrm{ml}$ was used to define positive samples.
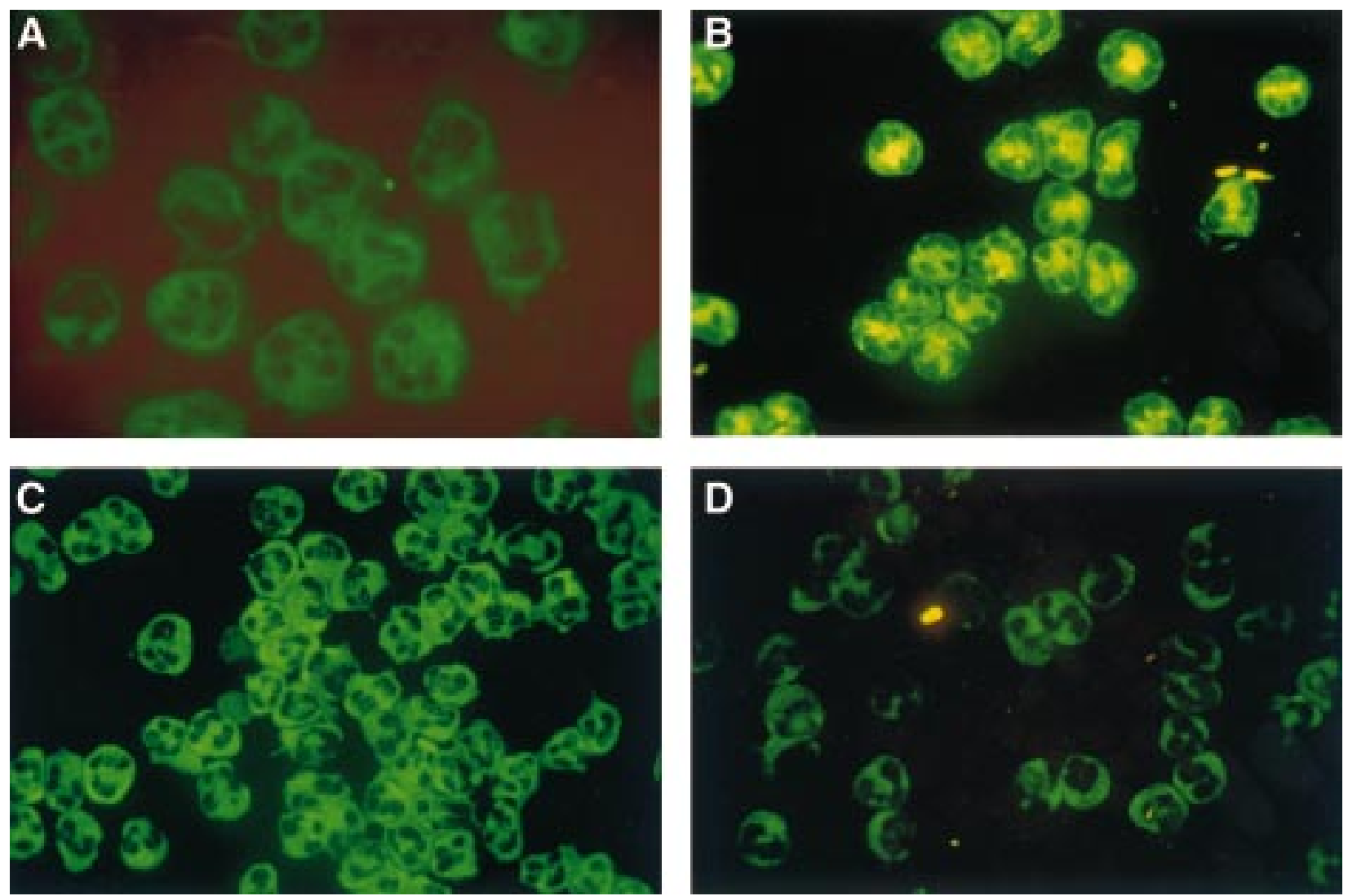

Figure 1 Ethanol fixed normal human neutrophil preparations with fluorescein isothiocyanate conjugated rabbit antihuman IgG. (A) Royal College of Pathologists of Australasia (RCPA) quality assurance programme (QAP) specimen: no evidence of centrallinterlobular accentuation of cytoplasmic staining. (B) "True" C-ANCA pattern: granular cytoplasmic staining with central/interlobular accentuation. ${ }^{20}$ (C) "Flat" ANCA pattern: homogeneous granular cytoplasmic staining with no central/interlobular accentuation. ${ }^{10}$ (D) "Atypical" ANCA pattern: one of the other cytoplasmic staining patterns not falling into categories $(B)$ or $(C)$. 
Table 1 Antineutrophil cytoplasmic antibody (ANCA) indirect immunofluorescence pattern versus PR3-ANCA ELISA results

\begin{tabular}{|c|c|c|c|}
\hline \multirow{2}{*}{$\begin{array}{l}\text { Cytoplasmic } \\
\text { ANCA } \\
\text { IIF pattern }\end{array}$} & \multicolumn{3}{|c|}{ PR3-ANCA ELISA } \\
\hline & $\begin{array}{l}\text { ELISA } \\
\text { positive }\end{array}$ & $\begin{array}{l}\text { ELISA } \\
\text { negative }\end{array}$ & Totals \\
\hline True C-ANCA & $25(34.5 \%)^{\star}$ & $2(3 \%)$ & $27(37.5 \%)$ \\
\hline Flat & $0(0 \%)$ & $21(29 \%)$ & $21(29 \%)$ \\
\hline Atypical & $1(1.5 \%)$ & $23(32 \%)$ & $24(33.5 \%)$ \\
\hline Totals & $26(36 \%)$ & $46(64 \%)$ & 72 \\
\hline
\end{tabular}

*True C-ANCA IIF pattern was significantly correlated with the presence of PR3-ANCA by ELISA $\left(\mathrm{p}<0.0001, \chi^{2}\right.$ and Fisher's exact tests).

ELISA, enzyme linked immunosorbent assay; IIF, indirect immunofluorescence.

Table 2 Antineutrophil cytoplasmic antibody (ANCA) indirect immunofluorescence patterns versus presence of systemic vasculitis (including Wegener's granulomatosis)

\begin{tabular}{ll}
\hline $\begin{array}{l}\text { Cytoplasmic ANCA IIF } \\
\text { pattern }\end{array}$ & $\begin{array}{l}\text { Number of cases with systemic } \\
\text { vasculitis (including WG) }\end{array}$ \\
\hline True C-ANCA & $20 / 23(87 \%)^{\star} \dagger$ \\
Flat & $0 / 19(0 \%)^{\star} \ddagger$ \\
Atypical & $6 / 22(27 \%)^{\dagger \dagger}$ \\
All cytoplasmic & $26 / 64(41 \%)$ \\
\hline
\end{tabular}

*True C-ANCA $v$ flat: $\mathrm{p}<0.0001$ (Fisher's exact test). $\dagger$ True C-ANCA $v$ atypical: $\mathrm{p}<0.0001$ (Fisher's exact test). $\$$ Flat $v$ atypical: $\mathrm{p}=0.023$ (Fisher's exact test).

IIF, indirect immunofluorescence; WG, Wegener's granulomatosis.

Six $(28.5 \%)$ of the "flat" ANCA sera were tested for BPI-ANCA by ELISA (ORGenTec) using the same technique as for the PR3ANCA ELISA.

CLINICAL DATA

The patients' underlying diagnoses were ascertained by reviewing the case records if these were held at Westmead Hospital, or by discussion with the requesting doctor. The 1994 Chapel Hill Consensus Conference definitions for Wegener's granulomatosis and other vasculitic entities were used for the diagnosis and classification of patients. ${ }^{16} \mathrm{~A}$ positive ANCA result was not used as a criteria for diagnosing systemic vasculitis or Wegener's granulomatosis.
STATISTICAL ANALYSIS

The $\chi^{2}$ test was used for comparison of results between groups, and Fisher's exact test where appropriate.

\section{Results}

“TRUE" C-ANCA PATTERNS AND PR3-ANCA POSITIVITY

Of the 72 specimens, 27 (37.5\%) showed a true C-ANCA pattern, 21 (29\%) a flat ANCA, and $24(33.5 \%)$ an atypical ANCA (table 1). There was a strong association between the true C-ANCA pattern and PR3-ANCA by ELISA. Thus 25 of 27 specimens with a true C-ANCA $(92.5 \%)$ contained PR3-ANCA, compared with none of 21 with a flat ANCA and only one of 24 with an atypical ANCA ( $p<0.0001)$.

The median and mode titres of all three patterns were the same at $1: 160$, but there was a trend towards higher true C-ANCA titres.

\section{“TRUE" C-ANCA PATTERNS AND SYSTEMIC} VASCULITIS

Clinical information was obtained for 23 of 27 sera $(85 \%)$ with a true C-ANCA, 19 of 21 sera $(90.5 \%)$ with a flat ANCA, and 22 of 24 sera (91.5\%) with an atypical ANCA (table 2). Of these 64 patients, only $26(41 \%)$ had features consistent with systemic vasculitis (including Wegener's granulomatosis) and tissue biopsy evidence of vasculitis. However, of the 23 sera with a true C-ANCA pattern, $20(87 \%)$ had a diagnosis of systemic vasculitis (including Wegener's granulomatosis), increasing the positive predictive value of the true C-ANCA pattern to $87 \%$. One of the two patients with a true C-ANCA pattern (1:640 titre) but negative PR3-ANCA (confirmed on a different PR3-ANCA ELISA kit (Euro-Diagnostica)) presented with pulmonary haemorrhage and acute renal failure and had renal biopsy findings consistent with Wegener's granulomatosis. No clinical data were available for the other patient.

In contrast, none of 19 patients with a flat ANCA had evidence of systemic vasculitis

Table 3 "Flat" antineutrophil cytoplasmic antibody (ANCA) patterns: clinical data and BPI-ANCA results

\begin{tabular}{|c|c|c|}
\hline Titre & Clinical data & $B P I-A N C A$ \\
\hline $1: 160$ & Cutaneous leucocytoclastic vasculitis. No evidence of systemic vasculitis/WG & Not done \\
\hline $1: 160$ & Benign intrahepatic cholestasis. No evidence of systemic vasculitis/WG & Positive \\
\hline $1: 160$ & Non-specific duodenitis. No evidence of systemic vasculitis/WG & Not done \\
\hline $1: 40$ & Ischaemic colitis. No evidence of systemic vasculitis/WG & Not done \\
\hline $1: 40$ & Inflammatory bowel disease, probably ulcerative colitis. No evidence of systemic vasculitis/WG & Positive \\
\hline $1: 160$ & Splenomegaly and lymphadenopathy. No evidence of systemic vasculitis/WG or malignancy & Not done \\
\hline $1: 160$ & $\begin{array}{l}\text { Bilateral upper quadrant abdominal pain. Upper abdominal ultrasound and gastroscopy normal. } \\
\text { No evidence of systemic vasculitis/WG }\end{array}$ & Not done \\
\hline $1: 160$ & Bacterial chest infection. No evidence of systemic vasculitis/WG & Not done \\
\hline $1: 160$ & AIDS, pneumocystis carinii pneumonitis. No evidence of systemic vasculitis/WG & Positive \\
\hline $1: 40$ & Postinfective left pleural effusion. No evidence of systemic vasculitis/WG & Not done \\
\hline $1: 40$ & Aspiration pneumonitis. Multiple pulmonary emboli. No evidence of systemic vasculitis/WG & Negative \\
\hline $1: 40$ & Acute rheumatic fever with cardiac involvement. No evidence of systemic vasculitis/WG & Not done \\
\hline $1: 160$ & Chronic tubulo-interstitial renal disease. No evidence of systemic vasculitis/WG & Positive \\
\hline $1: 160$ & Acute tubular necrosis followed by chronic renal failure. No evidence of systemic vasculitis/WG & Not done \\
\hline $1: 160$ & Peripheral neuropathy of unknown cause. No evidence of systemic vasculitis/WG & Not done \\
\hline $1: 160$ & $\begin{array}{l}\text { Autoimmune thyroiditis and haemolytic anaemia. Multiple autoantibodies. No evidence of } \\
\text { systemic vasculitis/WG }\end{array}$ & Negative \\
\hline $1: 160$ & $\begin{array}{l}\text { Persistent iritis. No evidence of inflammatory bowel disease, systemic vasculitis/WG, or other } \\
\text { connective tissue disease }\end{array}$ & Not done \\
\hline $1: 160$ & Septicaemia. Atherosclerotic ileal infarction. No evidence of systemic vasculitis/WG & Not done \\
\hline $1: 40$ & Probable thromboangiitis obliterans. No evidence of systemic vasculitis/WG & Not done \\
\hline $1: 40$ & No clinical information & Not done \\
\hline $1: 160$ & No clinical information & Not done \\
\hline
\end{tabular}


$(\mathrm{p}<0.0001 v$ true C-ANCA), although one (1:160 titre) had a cutaneous leucocytoclastic vasculitis (table 3 ).

Six of the 22 patients $(27 \%)$ with an atypical ANCA (titres of $1: 160$ to $>1: 640$ ) had a diagnosis of systemic vasculitis $(\mathrm{p}<0.0001 v$ true C-ANCA). The one patient with an atypical ANCA (1:160 titre) and a positive PR3-ANCA (confirmed on a different PR3-ANCA ELISA kit (Euro-Diagnostica)) had severe ulcerative colitis requiring pancolectomy, but had no evidence of vasculitis in the excised colon or systemic vasculitis elsewhere. This patient's serum was MPO-ANCA negative by ELISA (ORGenTec).

BPI-ANCA ELISA RESULTS

Of the six flat ANCA sera tested for BPIANCA (table 3), four were positive while two were negative.

\section{Discussion}

The diagnostic value of the "classical/true" C-ANCA IIF pattern has been confirmed by Rao et al in a meta-analysis in which C-ANCA had $98-99.5 \%$ specificity for Wegener's granulomatosis, ${ }^{4}$ and in a prospective study ${ }^{5}$ in which C-ANCA had 96\% specificity for Wegener's granulomatosis. The diagnostic value of the PR3-ANCA ELISA has also been demonstrated by Jennings et $a l,^{8}$ who detected PR3-ANCA in 10 of 13 patients with generalised Wegener's granulomatosis, two of 10 patients with microscopic polyangiitis, and three of six with segmental necrotising glomerulonephritis. In the recent European/ British cooperative ANCA assay standardisation study, PR3-ANCA had $68 \%$ sensitivity for Wegener's granulomatosis (97 patients), and specificities of $87 \%$ and $98 \%$ in disease and healthy controls respectively (Hagen EC et al, submitted for publication).

Baslund et al have reported that in 14 sera from patients with active Wegener's granulomatosis, a standard direct binding ELISA detected PR3-ANCA in 10 sera (71\%), but IIF was positive in 11 sera $(79 \%)$, and a combination of three capture PR3-ANCA ELISA was positive in 12 sera $(86 \%) .^{9}$ They concluded that IIF may be more sensitive for ANCA than the standard ELISA, hypothesising that PR3 may be denatured during coating onto the ELISA plate, resulting in the loss of conformational epitopes that are targeted by some ANCA. ${ }^{17}$ These conclusions may explain why one of our patients with biopsy proven Wegener's granulomatosis was true C-ANCA positive but PR3-ANCA negative.

We also found one patient with an atypical ANCA pattern, a positive PR3-ANCA, severe ulcerative colitis, but no evidence of systemic vasculitis. This is consistent with a report in which one of 15 patients with chronic ulcerative colitis but no evidence of systemic vasculitis had a positive PR3-ANCA. ${ }^{18}$

It is important that the true C-ANCA IIF pattern be strongly predictive for positive PR3ANCA results and systemic vasculitis (including Wegener's granulomatosis), even though ANCA should be only used as an adjunct to tissue diagnosis in the diagnosis of systemic vasculitis. ${ }^{19}$ However, in clinical practice tissue samples may be difficult to obtain or the rate of clinical deterioration may be such that urgent therapeutic decisions need to be made, based on serological tests alone. Furthermore, IIF results are typically available before confirmatory PR3-ANCA ELISA results, and therefore often strongly influence these decisions.

The diagnostic importance of IIF might be questioned given that PR3-ANCA ELISA are readily available, less prone to observer error, and have high diagnostic accuracy. However, IIF is likely to remain the preferred screening test for ANCA because (1) it also detects perinuclear ANCA associated with MPO-ANCA specificity and microscopic polyangiitis ${ }^{20}$; (2) IIF is cheaper to perform, particularly with an urgent specimen where the material costs of an ELISA for a single specimen exceed that of IIF several fold; and ( 3 ) because IIF may be more sensitive than the standard PR3-ANCA ELISA for Wegener's granulomatosis. ${ }^{9}$ Although a combination of capture PR3-ANCA ELISA was the most sensitive test for Wegener's granulomatosis, ${ }^{9}$ these assays are not widely available.

The antigenic specificity of the "flat" ANCA IIF pattern is of interest, given the description by Zhao et al of the novel neutrophil cytoplasmic antigen, bactericidal/permeability increasing protein (BPI). ${ }^{21}$ The investigators detected BPI-ANCA in 45 of 100 sera $(45 \%)$ that were negative for PR3-ANCA and MPO-ANCA. Although 38 of these 45 sera $(84.5 \%)$ were reported to be positive for C-ANCA, the precise staining patterns were not described. In our study, only four of the six flat ANCA sera (67\%) tested for BPI-ANCA were positive, indicating that BPI-ANCA are not the only cause of flat ANCA patterns. In particular, antibodies to other neutrophil cytoplasmic antigens (including azurocidin), and other cytoplasmic autoantibodies (for example, antismooth muscle antibodies) can produce a flat ANCA pattern. ${ }^{22}$

CONCLUSION

We recommend that the term "C-ANCA" be restricted to IIF patterns showing granular cytoplasmic staining with central or interlobular accentuation. All other cytoplasmic patterns including "flat" patterns should be reported as "C-ANCA (atypical)" until more is known about their antigen specificities and disease associations. This practice will result in fewer disparities between C-ANCA IIF and PR3ANCA ELISA results, greater diagnostic accuracy for systemic vasculitis (including Wegener's granulomatosis), and thus less confusion for requesting clinicians.

We wish to thank the Royal College of Pathologists of Australasia (RCPA) quality assurance programme (QAP) Board for sia (RCPA) quality assurance programme (QAP) Board for
allowing us to publish figure $1 \mathrm{~A}$ and report the results on this specimen.

1 Roberts DE. Antineutrophil cytoplasmic autoantibodies. Clin Lab Med 1992;12:85-98.

2 Rasmussen N, Wiik A. Indirect immunofluorescence examination for IgG-ANCA in sera submitted for the 1st international workshop on ANCA, 1988. APMIS 1989; national workshop
97(suppl 6):16-20. 
3 Rasmussen N, Wiik A. Autoimmunity in Wegener's granulomatosis. Immunobiology, autoimmunity and transplantation in otorhinolaryngology. In: Veldman JE, McCabe BF, Houzing EH, et al, eds. Proceedings of the 1st Inter-
national Conference, Utrecht, The Netherlands, 1984. national Conference, Utrecht, The Netherland

4 Rao JK, Weinberger M, Oddone EZ, et al. The role of antineutrophil cytoplasmic antibody (c-ANCA) testing in the diagnosis of Wegener granulomatosis. A literature review and meta-analysis. Ann Intern Med 1995;123:92532.

5 Rao JK, Allen NB, Feussner JR, et al. A prospective study of antineutrophil cytoplasmic antibody (c-ANCA) and clinical criteria in diagnosing Wegener's granulomatosis. Lancet 1995;346:926-31.

6 van der Woude FJ, Rasmussen N, Lobatto S, et al. Autoantibodies against neutrophils and monocytes: tool for diagnosis and marker of disease activity in Wegener's diagnosis and marker of disease

7 Goldschmeding R, van der Schoot CE, ten Bokkel Huinink $\mathrm{D}$, et al. Wegener's granulomatosis autoantibodies identify $\mathrm{D}$, et al. Wegener's granulomatosis autoantibodies identify lysosomes of normal human neutrophils. $\mathcal{F}$ Clin Invest $1989 \cdot 84: 1577-87$

8 Jennings JG, Chang L, Savige JA. Anti-proteinase 3 antibodies, their characterization and disease associations. Clin Exp Immunol 1994;95:251-6.

9 Baslund B, Segelmark M, Wiik A, et al. Screening for antineutrophil cytoplasmic antibodies (ANCA): is indirect immunofluorescence the method of choice? Clin Exp Immunol 1995;99:486-92.

10 Lock RJ. ACP Broadsheet No 143: January 1994. Detection of autoantibodies to neutrophil cytoplasmic antigens. $7 \mathrm{Clin}$ Pathol 1994;47:4-8.

11 Zola H, Roberts-Thomson P, McEvoy R. Autoimmune serology. In: Zola H, Roberts-Thomson P, McEvoy R, eds. Diagnostic immunopathology: laboratory practice and clinical application. Cambridge: Cambridge University Press, 1995: application.
12 Nassberger L. Distribution of antineutrophil cytoplasmic autoantibodies in SLE patients with and without renal autoantibodies in SLE patients with and
involvement. Am f Nephrol 1996;16:548-9.

13 Papo M, Quer JC, Pastor RM, et al. Antineutrophil cytoplasmic antibodies in relatives of patients with inflammatory bowel disease. Am f Gastroenterol 1996;91:151215.

14 Niles JL. Antineutrophil cytoplasmic antibodies in the classification of vasculitis. Annu Rev Med 1996;47:303-13.

15 Wiik A. Delineation of a standard procedure for indirect immunofluorescence detection of ANCA. APMIS 1989; 97(suppl 6): 12-13

16 Jennette JC, Falk RJ, Andrassy K, et al. Nomenclature of systemic vasculitides: proposal of an international consensus conference. Arthritis Rheum 1994;37:187-92.

17 Bini P, Gabay JE, Teitel A, et al. Antineutrophil cytoplasmic autoantibodies in Wegener's granulomatosis recognize conformational epitope(s) on proteinase 3.7 Immunol 1992;149:1409-15.

18 Aitola P, Miettinen A, Mattila A, et al. Effect of proctocolectomy on serum antineutrophil cytoplasmic antibodies in patients with chronic ulcerative colitis. I Clin Pathol 1995;48:645-7.

19 Savage COS, Harper L, Adu D. Primary systemic vasculitis. Lancet 1997;349:553-8.

20 Falk RJ, Jennette JC. Anti-neutrophil cytoplasmic autoantibodies with specificity for myeloperoxidase in patients with systemic vasculitis and idiopathic necrotizing glomerulonesystemic vasculitis and idiopathic necrotidn
phritis. $N$ Engl f Med 1988;318:1651-7.

21 Zhao MH, Jones SJ, Lockwood CM. Bactericidal/ permeability-increasing protein (BPI) is an important antigen for anti-neutrophil cytoplasmic autoantibodies ANCA) in vasculitis. Clin Exp Immunol 1995;99:49-56.

22 Savige JA, Paspaliaris B, Silvestrini R, et al. A review of immunofluorescent patterns associated with antineutrophil cytoplasmic antibodies (ANCA) and their differentiation from other antibodies. 7 Clin Pathol 1998;51:569-75. 